\title{
Prospects for improved bluetongue vaccines
}

\section{Polly Roy, Mark Boyce and Robert Noad}

\section{Abstract | Bluetongue has been recognized as a viral disease of livestock for more than} 100 years. Repeated incursions of Bluetongue into Europe since 1998 have been particularly devastating for highly sensitive European fine-wool sheep breeds, and have resulted in a resurgence of interest in vaccine manufacture. Fortunately, the virus and its serology are well understood and vaccination prevents the disease. However, current vaccines are not without their problems, and many new approaches are being tested to improve the safety and breadth of protection afforded. This Review describes the leading technologies for improved bluetongue vaccines and looks ahead to how advances in other viral vaccines might be applied to this disease.

\section{Serotype}

A group of closely related

microorganisms that are

distinguished by a

characteristic set of antigens.

Bluetongue virus serotypes

are defined by the

neutralization of virus

infectivity by serum antibody.
Department of Infectious \& Tropical Diseases, London School of Hygiene \& Tropical Medicine, Keppel Street, London WC1E 7HT, UK. Correspondence to P.R. e.mail:Polly.Roy@lshtm.ac.uk doi:10.1038/nrmicro2052
Bluetongue was first recognized when European finewool breeds of Merino sheep were imported into South Africa in 1900 (REFS 1,2). The disease spread rapidly throughout Africa and subsequently to many other countries beyond the African continent. In susceptible sheep flocks, mortality for infected animals is between 50 and $70 \%^{3,4}$, and, as the disease has been reported in almost every continent, with the exception of Antarctica, bluetongue is one of the most widespread animal pathogens. Symptoms of bluetongue disease are largely the result of damage to small blood vessels, and include oral ulceration, facial and pulmonary oedema, vascular thromboses and necrosis of infected tissues ${ }^{5}$ (BOX 1). In sheep, the onset of the disease is typically marked by fever that lasts approximately 5-7 days, after which distinctive lesions appear in the mouth, accompanied by excessive salivation. The tongue can also be severely affected, occasionally turning blue. In contrast to sheep, infected cattle experience prolonged viraemia, and infection during pregnancy can often cause teratogenic defects in calves and abortion of the fetus ${ }^{6-10}$. Bluetongue is a non-contagious viral disease spread by biting midges of the Culicoides genus (BOX 1). Primary control measures are therefore based on the control of vector insects in the affected area (see Further information for a link to the World Organisation for Animal Health (bluetongue diseases data) and the National Farmers Union). However, because the virus replicates in both the insect vector and in a range of other ruminants, including cattle and goats, often with prolonged viraemia and less-severe disease symptoms, it is difficult to control the virus using these measures once it becomes established.
Bluetongue virus (BTV) is endemic in many tropical and sub-tropical countries, and until recently, outbreaks of bluetongue disease in Europe, primarily in southern European countries, were sporadic and rare. However, since 1998, there have been separate and repeated incursions of bluetongue into Europe and 6 of the 24 different serotypes (BTV-1, -2, -4, -8, -9 and -16) have been introduced into mainland Europe ${ }^{11}$. Early outbreaks in southern Europe were the result of separate incursions from the Middle East into Greece and the Balkans, and from North Africa (Tunisia, Algeria and Morocco) into Spain, Corsica, Sardinia and the Balearic Islands ${ }^{12}$. A later outbreak of BTV-8 in northern Europe, which started in 2006 and spread as far north as France, Belgium, Holland and Germany in 2006, to the United Kingdom in 2007 and to Sweden in 2008, was the result of a separate introduction of the virus. BTV-8 is most similar to a strain found in subSaharan Africa ${ }^{13}$. However, whether this incursion was the result of the movement of infected animals or the movement of the insect vector that transmits the virus between animals is still hotly debated ${ }^{14}$. Climate change could have contributed to the emergence of bluetongue in Europe, through the increased distribution and size of insect vector populations ${ }^{15}$. However, an additional serotype, BTV-6, that had not previously been seen in Europe, was detected in October 2008 in sheep in the Netherlands and was confirmed as a live attenuated vaccine strain (see Further information for a link to The Center for Food Security and Public Health (animal disease information)), implying that the source was imported livestock. 


\section{Box 1 | Transmission and replication of bluetongue virus}

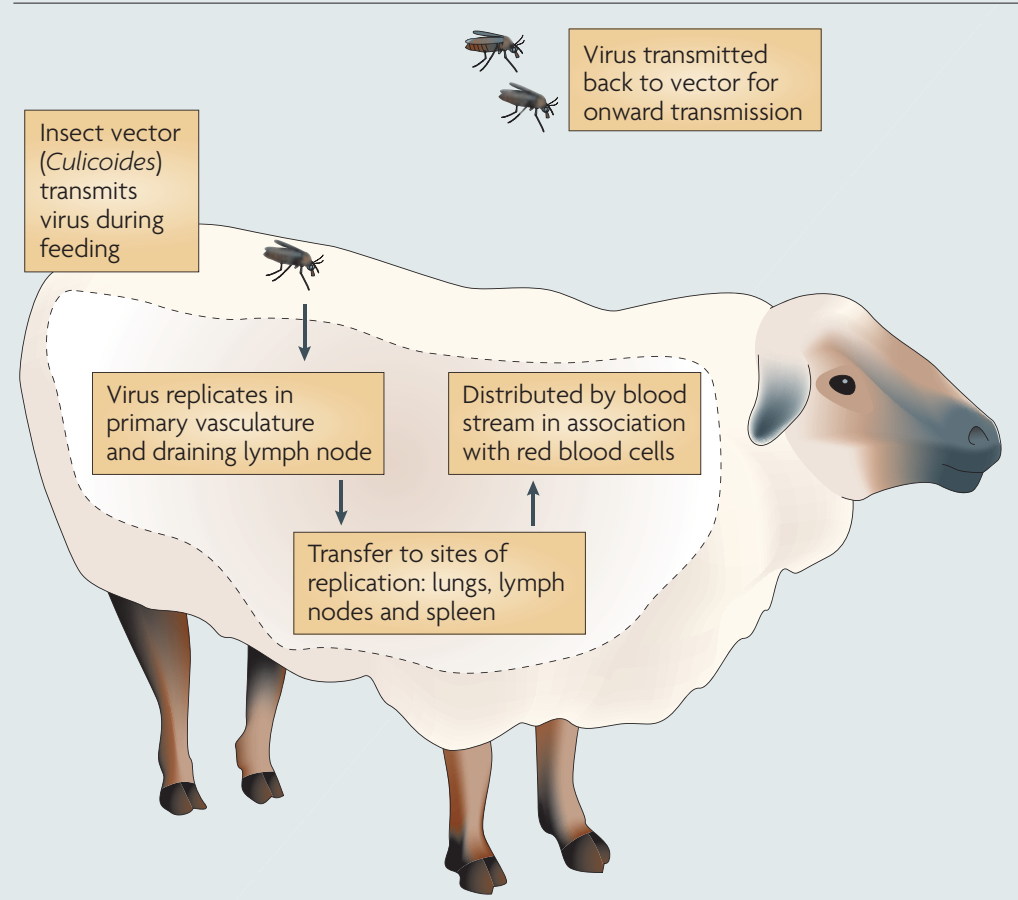

Bluetongue virus (BTV) replicates in both wild and domestic ruminants, including some species of deer, which makes mass vaccination of domestic livestock an incomplete measure for immunization of the susceptible host population. BTV replicates in both the mammalian host and the Culicoides insect vector. Transmission to the insect vector occurs when a female insect takes a blood meal from an infected mammal. The virus is transmitted to naive ruminants when the insect with infected salivary glands feeds again.
Segmented virus

A virus in which the genome is divided into two or more physically distinct molecules, which are packaged into a single virus particle.

\section{Virus structure and vaccine challenge}

BTV, the causative agent of bluetongue disease, is well defined at both the structural and molecular levels. BTV is a member of the Reoviridae family, a widely distributed group of viruses that infect humans and other mammals (rotaviruses, orbiviruses, seadornaviruses and orthoreoviruses), fish (aquareoviruses), birds (orthoreoviruses), insects (cypoviruses and idnoreoviruses), plants (oryzaviruses, fijiviruses and phytoreoviruses) and fungi (mycoreoviruses). The BTV genome consists of ten segments of doublestranded RNA (dsRNA) that each encode a different viral protein and a multilayered protein capsid, but is not surrounded by a lipid envelope. BTV particles are architecturally complex structures (FIG. 1) that are organized into three shells which form the bulk of the virus particle. The inner shell is composed of 120 copies of VP3 and contains minor amounts of 3 enzymatic proteins (VP1, VP4 and VP6) that are located at the five-fold symmetry axes of the particle. The middle shell is composed of 780 copies of VP7 that are arranged as 260 trimers. Such double-layered core particles serve as a foundation for the two remaining major structural proteins, VP2 and VP5, which together form the outer shell. A total of 180 molecules of VP2 are arranged as 60 surface spikes, and these spikes are responsible for attaching the virus to the cell surface, whereas 360 molecules of VP5 form 120 globular-shaped structures that facilitate cell-membrane penetration $^{16-18}$. Together, VP2 and VP5 form a continuous layer around the core, yielding a well-ordered morphology of the virus. These two proteins are highly variable among the different serotypes, although close phylogenetic relationships were easily detectable, indicating that mutations may have played a major part in generating multiple serotypes ${ }^{18}$.

BTV serotype is based on serological neutralization of virus particles rather than on sequence variation. In general, antibodies to one serotype do not crossneutralize virus from another serotype. However, in vaccinated sheep, there is some evidence for at least partial cross-protection between vaccines that target closely related serotypes ${ }^{19}$. In terms of the immune response, there is evidence that both antibodies ${ }^{20}$ and T-cell-mediated responses ${ }^{21-23}$ can be protective. However, one report has found a closer correlation between $\mathrm{T}$-cell responses than neutralizing antibody responses to protection from virulent virus ${ }^{23}$.

Like other segmented viruses, reassortment of genome segments in hosts co-infected with more than one strain of BTV can readily lead to progeny strains with a mixture of the characteristics of the parental strain $^{24-31}$ (FIG. 2). In one study, 48 progeny viruses from cells co-infected with 2 parental strains of BTV with different dsRNA profiles were assessed, and 19 of these viruses $(\sim 40 \%)$ were found to be reassortants between the parental strains ${ }^{26}$. This is probably an underestimate of the true rate of reassortment because in this experiment only two of ten genomic dsRNA segments (S5 and S10) were used as markers for reassortment. Thus, an ideal vaccine for bluetongue would protect against as many virus serotypes as possible but would not revert to virulence, and would not recombine with circulating strains of the virus. In addition, given the dangers posed by the growth of large cultures of pathogenic virus for production of inactivated vaccines, any vaccine strain should ideally be unable to replicate in the field. Finally, an ideal BTV vaccine strain would pose no danger of replicating within insects and would be compatible with tests to distinguish between infected and vaccinated animals.

As BTV productively infects only ruminant species there is no small-animal model, and consequently all vaccine trials must use large animals housed in biocontainment facilities. The financial cost associated with trials of candidate BTV vaccines is therefore high. Only two vaccine types for bluetongue are currently available commercially: live attenuated vaccines and inactivated virus vaccines. Live attenuated bluetongue vaccines have a long history. An early report from South Africa stated that attenuated virus produced by serial passage of BTV in sheep could be used successfully as a vaccine ${ }^{32}$. Subsequently, South African scientists developed the first egg-adapted attenuated strains. This work led to the availability of attenuated virus vaccines for 15 different serotypes, which played a major part in control of the disease not only in South Africa but also in many other countries ${ }^{33}$. 


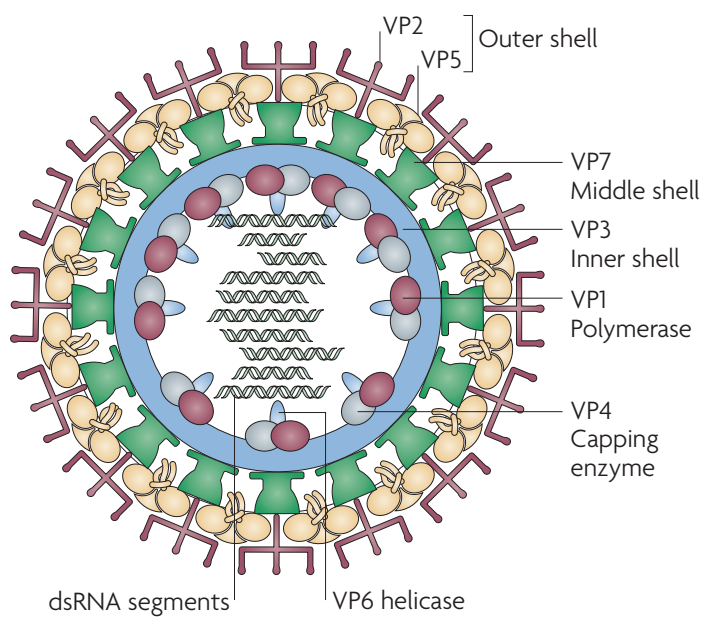

Figure 1 | Bluetongue virus morphology. The bluetongue virus particle consists of the double-stranded RNA (dsRNA) genome surrounded by three concentric protein layers. The viral genome is divided into ten linear dsRNA molecules, each of which encodes a single protein and is located at the centre of the particle. The viral genome is surrounded by the icosahedral inner protein layer, which consists of VP3. On the inner surface of this layer, at the five-fold symmetry axes of the particle, are the RNA-dependent RNA polymerase (VP1) and capping enzyme (VP4). A viral helicase (VP6) is probably also associated with the VP1-VP4-RNA complex. The VP3 layer is surrounded by VP7, a trimeric protein. Contacts between these two layers are formed by non-specific hydrophobic interactions. The outer layer (which consists of VP2 and VP5) of the virus particle is responsible for attachment and penetration of target cells.

However, similar, live attenuated vaccines have also been developed in various countries in response to endemic serotypes or a particular outbreak ${ }^{33-37}$ (TABLE 1). Despite the apparent success of attenuated virus vaccines in temporarily controlling BTV in particular areas, their use is not without controversy. Teratological effects as a result of vaccination with attenuated BTV are well documented ${ }^{38-40}$. Indeed, by as early as 1955 , Schultz and Delay had shown that an egg-adapted BTV vaccine strain caused congenital malformations and fetal death in the lambs of ewes that were vaccinated in South Africa ${ }^{38}$. Nevertheless, the vaccine was considered safe by the manufacturer, and its use was continued in non-pregnant sheep in South Africa and other countries. A recent report further documented that, following vaccination in laboratory experiments, viraemia was sufficient for transmission of the vaccine strain to the insect vector ${ }^{36}$. This effect has also been observed in the field in Italy, where unvaccinated sentinel cattle and the insect vector were found to be positive for a vaccine strain of the virus ${ }^{41}$. Of particular concern is that the segmented nature of the BTV genome allows genes to be swapped between strains that co-infect the same animal ${ }^{42}$. This occurred in 2002 in Italy where a circulating BTV-16 strain was found to be a reassortment between BTV-2 and BTV-16 live attenuated vaccine strains ${ }^{43}$. Thus, attenuated vaccines for BTV may offer a route to control of the disease, but, owing to the possibility of teratological effects and the evidence that attenuated strains can be transmitted to non-vaccinated animals and swap genes with circulating field strains, they are not suitable for a programme designed to eradicate the disease.

More recently, inactivated vaccines have been prepared by treating BTV with beta-propiolactone ${ }^{44,45}$, gamma radiation ${ }^{46}$ or binary ethylenimine ${ }^{38,47,48}$. Inactivated vaccines based on these experiments are commercially available and have demonstrated good immunogenicity and safety ${ }^{49}$ (TABLE 1). In trials of commercially available bivalent vaccine in cattle, vaccination with 2 doses of bivalent vaccine 4 weeks apart was sufficient to protect calves from viraemia following challenge ${ }^{37}$. These vaccines were significantly more effective than the attenuated vaccines, provided inactivation was complete and quality control was rigorously implemented. However, it is important that the virus be completely inactivated in every vaccine batch, as otherwise vaccination could lead to some of the problems discussed above for attenuated vaccines. Furthermore, the vaccine requires two doses, which increases costs, and vaccine production involves the growth of large amounts of infectious virus before inactivation. There is currently a programme of mass vaccination of sheep and cattle in northern Europe in response to the ongoing outbreak of BTV-8.

\section{Experimental vaccines}

A number of potential alternatives have been investigated to address the unmet requirements for an effective bluetongue vaccine: low cost, ability to distinguish between vaccinated and infected animals, broad protective immunity against multiple serotypes and, preferably, a single dose. To be successful, a new vaccine will have to address the underlying problem of the generally poor immunogenicity of subunit vaccines. It was demonstrated over 20 years ago that the VP2 protein of BTV alone was sufficient to elicit protective immune responses in sheep ${ }^{50}$. Since then, VP2 alone, and in combination with other viral proteins, has been delivered to animals in laboratory-scale experiments to assess protective efficacy using a range of different approaches, including purified recombinant protein ${ }^{51,52}$, canarypox-vectored expression ${ }^{53}$, and capripox-vectored expression ${ }^{54}$. For the poxvirus-based systems, poxvirus is used as a vehicle for the transfer of BTV genes into sheep cells, where BTV proteins are then synthesized. Perhaps unsurprisingly, given the initial observation, the vaccination of sheep with canarypox vector that co-expressed VP2 and VP5 elicited neutralizing antibody and protected sheep against challenge with $\mathrm{BTV}^{53}$. The canarypox vaccine vector is a ubiquitous vector that is safe (as it does not replicate in mammals), stable, and able to induce both humoral and cell-mediated immune responses. A further alternative approach used a capripox vector to express VP2, VP7, and the nonstructural proteins NS1 and NS3, all of which partially protected sheep from the disease ${ }^{54}$. Thus, these vaccines have generally been effective when tested in sheep and
Relating to birth defects,

usually gross malformations. 


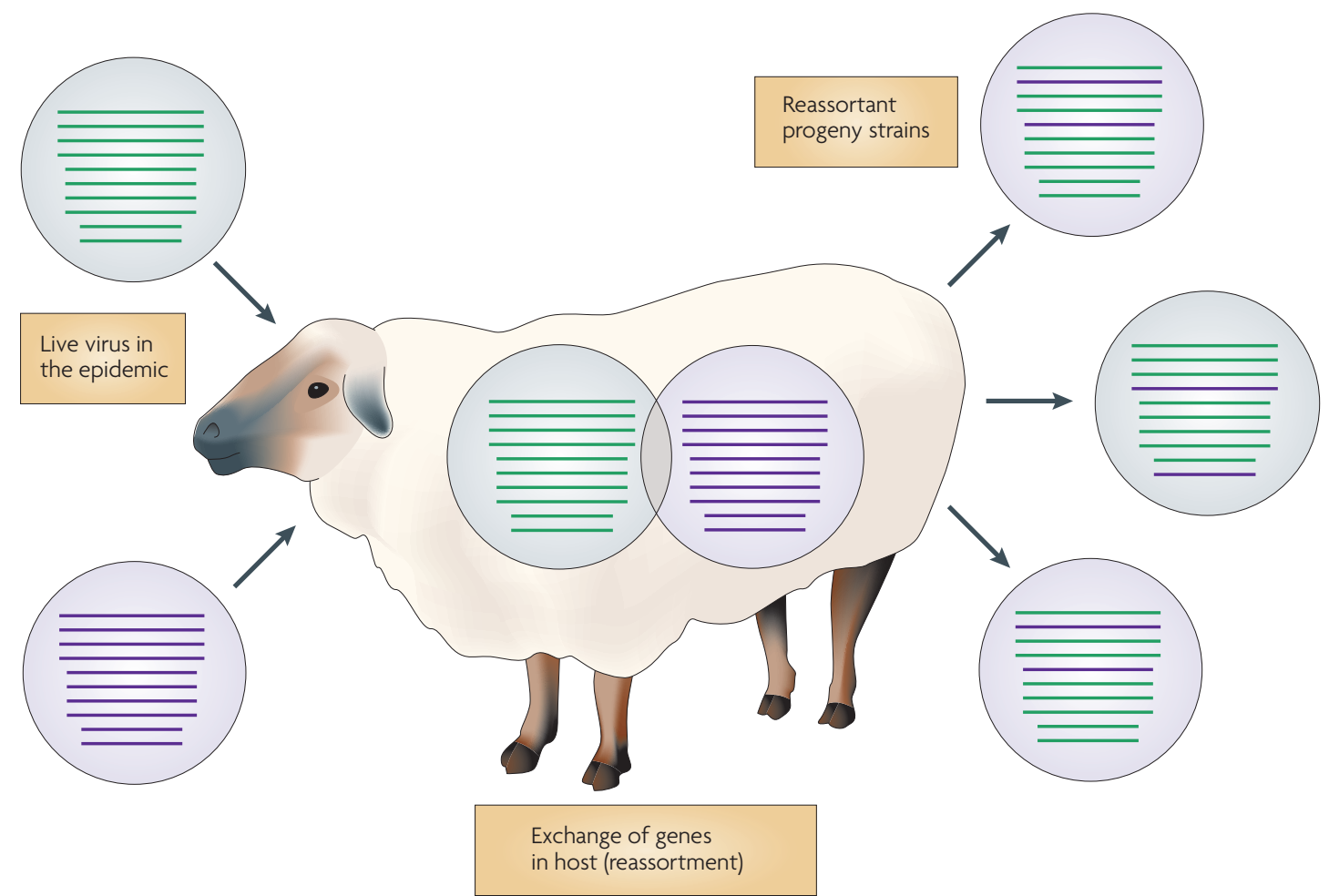

Figure 2| Reassortment. Like other viruses with segmented genomes, bluetongue virus genes are carried on separate pieces of RNA that are co-packaged into each infectious particle. When two different strains of BTV infect the same host, the animal acts as a mixing vessel for the pieces of RNA from the different parental viruses, and the progeny virions generated have genomes that are mixtures of these RNA segments. As RNA segments encode proteins that affect the serotype and virulence of the virus, progeny virions can be generated that have different characteristics than the original parental strains.

Virus-like particle

A particle that is structurally similar to an infectious virus particle, except that it lacks the viral genome and is therefore non-infectious. other ruminants, although it should be noted that the capripox vaccine was only partially protective, suggesting that not only the protein expressed but also the amount produced and its availability to interact with the immune system may be crucial. Importantly, these trials include systems such as baculovirus-based protein production and canarypox expression, which are already commercially viable and therefore offer realistic opportunities for improved vaccines for the virus.

One option that has been pioneered for the recombinant protein approach for BTV vaccines is the assembly of virus-like particles (VLPs). VLPs substantially improve immunogenicity compared with VP2only vaccines, because more of the VP2 antigen is in a correctly folded conformation and is presented to the immune system in an identical form, at a physical level, to the virus particle itself. Immunization trials with VLPs for BTV and other viruses have shown that VLPs elicit stronger and longer-lasting immune responses than unassembled subunit vaccines, and efficiently stimulate both B- and T-cell responses ${ }^{55,56}$. However, because the VLPs contain only the protein, and not the nucleic acid, component of the virus, there is no chance of reversion to virulence, reassortment or incomplete inactivation, which remain possible with other BTV vaccines. Similarly, poxvirus-vectored vaccines that express a small fraction of the BTV genes have no risk of acting as a source of virulent BTV, but retain the theoretical potential to recombine with field strains. Other VLP vaccines have recently reached the market for human papillomavirus (HPV) ${ }^{57-60}$, showing not only that the technology is feasible for scale up, but also that it represents a safe and effective alternative to traditional vaccines for viral diseases.

Like the new-generation HPV vaccines, VLPs for BTV are produced in insect cell culture using a baculovirus-based protein expression system. This eukaryotic expression system can produce large amounts of protein more efficiently than mammalian cell expression systems, it can fold and assemble proteins and large complexes (a single BTV VLP has a molecular mass of $\sim 83.8 \mathrm{mDa}$ ) and it uses an environmentally disabled form of an insect virus that is easily inactivated to drive protein expression ${ }^{61-63}$. Because the baculovirus used lacks a gene that is essential for the infection of its natural insect host, the recombinant virus cannot replicate in the environment. However, unlike HPV, which can form VLPs with only one of its two major structural proteins ${ }^{64}$, BTV is an architecturally more complex virus (FIG. 1). Of the seven structural BTV proteins, three are enzymatic and are involved in transcription of the virus genome ${ }^{18}$. Because these proteins do not seem to have a role in stabilization of the virus particle, and the VLP production procedure does not require infectious BTV at any stage, these proteins are omitted from the VLPs. VLPs are formed 


\begin{tabular}{|c|c|c|c|c|c|}
\hline & Bulgaria & France & Italy & Portugal & Spain \\
\hline \multicolumn{6}{|l|}{ Modified live virus } \\
\hline BTV-1 & Not used & Not used & 2007 & Not used & Not used \\
\hline BTV-3, $-8,-10$ and -11 & 1999-2000 & Not used & Not used & Not used & Not used \\
\hline BTV-2 & Not used & $2000-2002$ & 2002-2006 & Not used & $2000-2001^{\ddagger}$ \\
\hline BTV-4 & Not used & Not used & Not used & $2005-2006$ & 2004-2006 \\
\hline BTV-2 and -4 & Not used & 2003-2004 & 2004-2006 & Not used & $2003^{\S}$ \\
\hline BTV-2 and -9 & Not used & Not used & 2002-2006 & Not used & Not used \\
\hline BTV-16 & Not used & $2004 \|$ & Not used & Not used & Not used \\
\hline BTV-2, -4 and -16 & Not used & Not used & $2004^{\prime \prime}$ & Not used & Not used \\
\hline BTV-2, $-4,-9$ and -16 & Not used & Not used & $2004^{\prime \prime}$ & Not used & Not used \\
\hline BTV-2, -4 and -9 & Not used & Not used & $2005-2006$ & Not used & Not used \\
\hline BTV-9 & Not used & Not used & Not used & Not used & Not used \\
\hline \multicolumn{6}{|l|}{ Inactivated virus" } \\
\hline BTV-1 & Not used & Not used & Not used & 2007 & 2007 \\
\hline BTV-2 & Not used & 2004-2005 & Not used & Not used & Not used \\
\hline BTV-4 & Not used & $2004-2005$ & Not used & $2005-2007$ & 2005-2006 \\
\hline BTV-2 and -4 & Not used & $2005-2007$ & $2005-2007$ & Not used & Not used \\
\hline
\end{tabular}

by co-expression of the four major structural proteins of the virus (VP2, VP3, VP5 and VP7), which constitute the three shells of the virus particle.

To test whether it was possible to assemble full virus particles, it was first necessary to show that the core could be made in the absence of the three minor proteins and the dsRNA genome ${ }^{63}$. Recombinant baculoviruses that synthesized both VP3 and VP7 were isolated, and core-like particles (CLPs) were detected throughout the baculovirus-infected insect cells (BOX 2). The purified CLPs were similar in size and appearance to cores prepared from BTV. Subsequently, new baculovirus expression vectors were generated, allowing co-expression of four BTV structural proteins from a single recombinant virus in the same cell. These proteins were found to assemble into VLPs. Electron cryo-microscopy showed that the CLPs and VLPs were indistinguishable from the equivalent structures produced during a normal infection ${ }^{65-68}$. Furthermore, like authentic virus particles, VLPs had high levels of haemagglutination, and antibodies to VLPs produced in guinea pigs had high neutralization activity against infectious virus of the corresponding serotype ${ }^{69}$. To test if VLPs could elicit protective responses in sheep against BTV infection, groups of BTV-susceptible, 1 -year old naive Merino sheep were subcutaneously immunized with different concentrations of purified VLPs for BTV-10 and were given a booster 3 weeks later. Immune response was monitored by collecting neutralizing antibody titre in serum samples at regular intervals following vaccination. Sheep immunized with VLPs developed different levels of neutralizing antibodies depending on the amount of VLPs administered $^{70}$. Significant levels of neutralizing antibodies were elicited with all concentrations of VLPs and persisted throughout the study, and saline-immunized control sheep remained seronegative. All sheep were challenged by subcutaneous inoculation of $1 \mathrm{ml}$ of infective sheep blood that contained virulent virus 117 days after vaccination. To assess the disease status of the sheep, clinical reactions were assessed against a standardized clinical reaction index and viraemia was monitored from 3-14 days post-challenge ${ }^{70}$. VLPimmunized sheep developed neither clinical signs nor detectable viraemia, whereas the control sheep were viraemic and developed the high neutralizing antibody titres that are indicative of a primary infection. In these experiments, doses of BTV VLPs as low as $10 \mu \mathrm{g}$ were sufficient to afford protection from virulent virus challenge. This effect cannot simply be explained by the fact that VLPs contain VP2, as VP2 constitutes $23.88 \%$ of a virion by mass, and therefore $10 \mu \mathrm{g}$ of VLPs would contain no more than $2.39 \mu \mathrm{g}$ VP2. In experiments in which baculovirus that expressed VP2 alone was used to vaccinate sheep, 2 doses of $100 \mu \mathrm{g}$ each were needed to achieve complete protection in all vaccinated animals ${ }^{51,71}$. By contrast, vaccination with lower amounts of VP2 $(50 \mu \mathrm{g})$ did not protect all animals. Thus, VLPs could protect sheep from virulent virus challenge with 41 times less antigen than the vaccine that was composed of the recombinant subunit VP2 alone. This effect is in part due to the presence of other viral proteins, as $50 \mu \mathrm{g} \mathrm{VP2}$ was protective if co-administered with $25 \mu \mathrm{g}$ VP5 (REF. 51). However, this does not explain the full effect, and it is likely that the effectiveness of the VLP vaccine is based largely on the response of the immune system, which treats the particle as if it were a virus particle. 

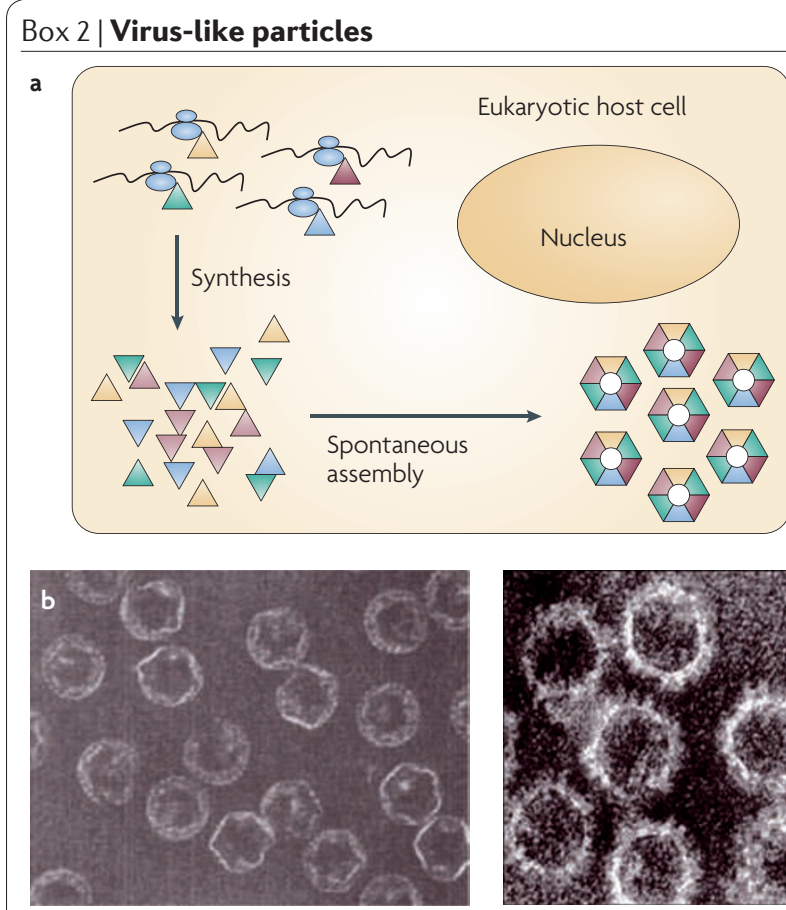

Inner smooth scaffolding layer (VP3 alone)

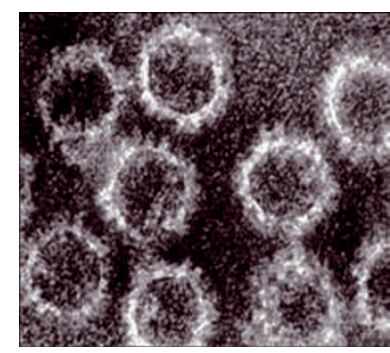

Double-layered particle (VP3 and VP7)
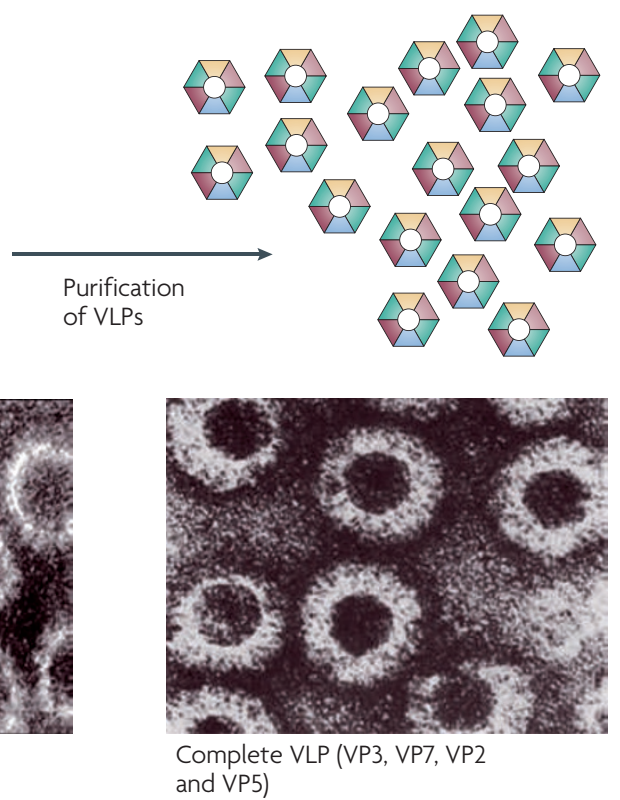

Virus-like particles (VLPs) are formed by the co-expression of virus structural proteins in the cytoplasm (see the figure, part a). The structural proteins of many viruses ${ }^{55} \mathrm{co}^{-a s s e m b l e ~ w i t h o u t ~ t h e ~ n e e d ~ f o r ~ a c t i v e ~ v i r u s ~ r e p l i c a t i o n . ~ F o r ~ b l u e t o n g u e ~}$ virus, it is possible to make VLPs in large quantities using the insect cell-based baculovirus expression system. Three distinct particles can be formed (see the figure, part b). The first is the inner smooth scaffolding layer, which is formed by a single BTV protein, VP3. The second is a double-layered particle that is composed of VP3 and is surrounded by VP7, forming a stable BTV core-like particle (CLP) with a regular spiky surface that corresponds to the trimers of VP7. The third particle is the complete VLP that contains all four major structural proteins (VP2 and VP5, which encapsidate the VP3-VP7 CLP), including the serotype-determining protein of the virus. This particle has a distinct roughened appearance under negative stain. VLPs contain only the antigenic proteins, and not the nucleic acid, components of the virus and therefore there is no possibility for reversion, recombination or reassortment.

To overcome the problem of serotype-specific protection, attenuated vaccines for BTV are often administered as cocktails that contain several virus serotypes. To test whether the VLP vaccine could similarly protect against multiple serotypes when administered as a mixture, VLPs for BTV-10 and BTV-17 were used to immunize sheep ${ }^{19}$. As in the study described above, 2 doses of either 10 or $50 \mu \mathrm{g}$ antigen were used per sheep. Both types of VLPs elicited neutralizing antibodies to the corresponding infectious virus. In addition, sheep vaccinated with a mixture of two types of VLPs produced antibodies that cross-neutralized infectious virus of a different serotype to the VLPs used in the immunization (BTV-4). In almost all cases, these neutralizing titres remained high throughout the 60-week period. Neutralizing antibody titres for the animals that received $50 \mu \mathrm{g}$ doses of VLPs were not significantly higher than those that received the $10 \mu \mathrm{g}$ doses. All the sheep were challenged 14 months after the booster vaccination by the subcutaneous injection of virulent $\mathrm{BTV}$ (BTV-4, BTV-10 or BTV-17). Animals challenged with virus that corresponded to the vaccine strains (BTV-10 and BTV-17), were completely protected and had no detectable viraemia or clinical reactions. In addition, animals that had been immunized with $50 \mu \mathrm{g}$ VLPs were substantially protected from BTV-4 infection ${ }^{19}$. VLPs are currently available for BTV- $1,-2,-4,-8,-10$ and -17 , and VLPs for other BTV serotypes are under construction.

In summary, VLPs afford long-lasting, type-specific protection from virulent BTV challenge. In addition, mixtures of VLPs for two different serotypes confer complete protection against both vaccine serotypes and partial protection against a related (based on the amino-acid sequence of VP2) non-vaccine serotype. Therefore, VLPs represent a valid approach for BTV vaccination. However issues need to be addressed, mainly in relation to the scale up of production to an industrial level and the fact that current formulations have only been tested in the two-dose per animal format. The current HPV VLP used in humans is expensive, but it is likely that these costs can be largely overcome for a veterinary vaccine. Indeed, one leading Dutch veterinary vaccine manufacturer (Intervet) is already marketing baculovirus-produced, recombinant protein antigens. 
Box 3 | Recovery of BTV from cDNA clones
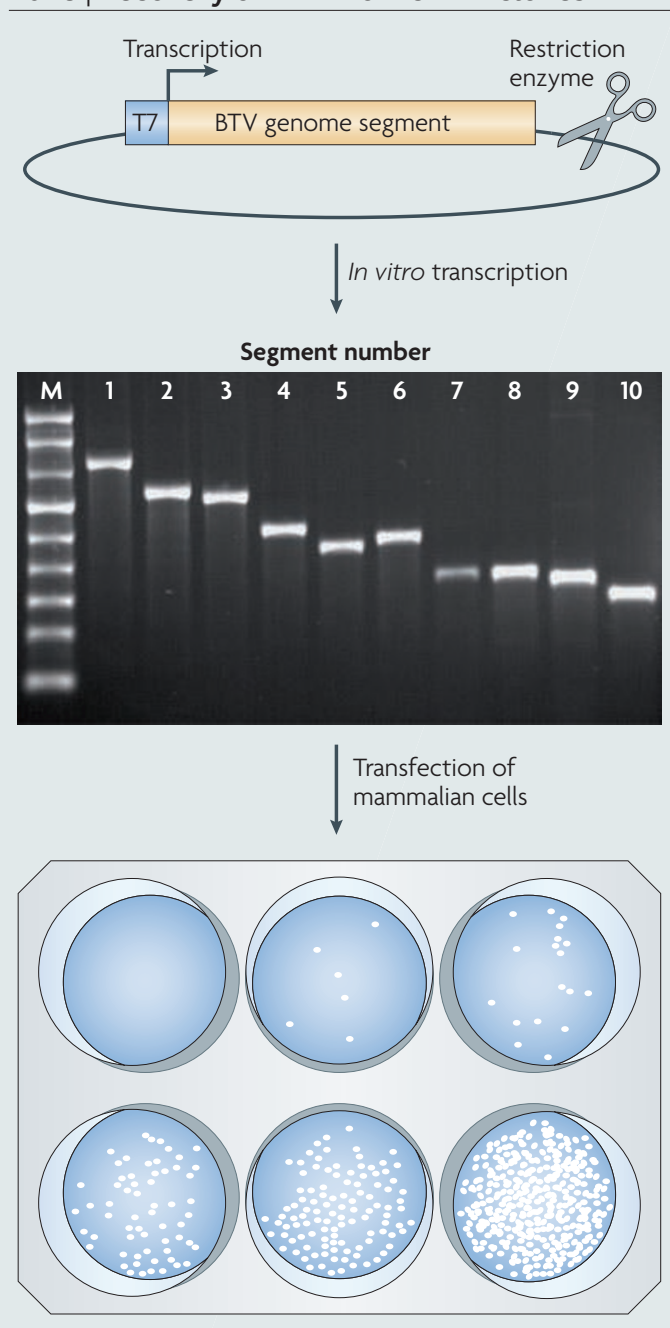

The steps needed to recover bluetongue virus (BTV) from cDNA are shown in the figure. T7 plasmids contain the full-length BTV genome segment flanked by a T7 phage promoter and a restriction enzyme site, which defines the BTV $3^{\prime}$ end sequence during transcription. Transcripts that correspond to each of the ten BTV segments are prepared in vitro by transcription from the T7 phage promoter, and their integrity is checked on $1 \%$ denaturing agarose gels. All ten transcripts are co-transfected into susceptible mammalian cells and overlaid with agarose. BTV plaques are picked and amplified using standard virological techniques. Using this approach, it is possible, for the first time, to introduce directed mutations into replicating virus, including those that selectively attenuate the virus ${ }^{72}$. M, molecular weight marker.

\section{Reverse genetics and future vaccines}

Traditional live vaccines for BTV rely on the attenuation of virus by passage in eggs or sheep. However, the mutations that define this attenuation are undocumented. The recent development of a reverse genetics system for BTV makes possible the rational design of attenuated vaccines (BOX 3). Infectious BTV is produced entirely from DNA clones by generating one transcript in vitro for each genome segment, and using these transcripts to transfect permissive cells ${ }^{72}$. This system allows the introduction of any mutation into the genome of BTV, as long as the resulting virus is viable. The ability to test the virulence of BTV mutants in the ruminant host will allow the identification of the pathogenicity determinants of BTV, and these results can be used to inform the design of vaccine strains with multiple attenuating mutations. The risk of reversion to virulence in a vaccinated population can be substantially reduced by introducing more than one attenuating mutation into the genome of the engineered strain. The possibility of regenerating a virulent BTV strain through genome segment reassortment with wild-type strains can be reduced when the attenuating mutations are present on several genome segments. Reverse genetics data and the formation of BTV VLPs have confirmed that outer capsid proteins from phylogenetically diverse serotypes can assemble on the conserved core proteins to create viable BTV strains ${ }^{69,72}$. This observation suggests that it will be possible to use a defined attenuated genetic background and introduce the antigenically important outer capsid proteins from the serotypes of interest. This will be relevant in regions where protection from several co-circulating strains is needed, such as mainland Europe. The 'strain-by-strain' testing of each candidate vaccine strain for efficacy and safety can be streamlined as each new strain generated contains the same genetic background as previously tested strains.

Reverse genetics also provides a basis for the development of disabled infectious single cycle (DISC) vaccines for BTV, which allows the virus to infect the vaccinated animal, but stops it from completing a replication cycle (FIG. 3). The resulting aborted infection allows the expression of viral proteins at natural sites of infection without the production of infectious virus or disease in the animal, and can be considered to be an extreme form of attenuation. To produce a DISC strain, an essential gene must be deleted from the viral genome, and a complementing cell line must be produced that contains the deleted gene. Together, the complementing cell line and the defective viral genome produce a complete set of viral proteins, allowing the replication of the virus. In any other cell, such as the natural host, the viral genome is defective because the essential viral gene product is not expressed. A complementing cell line has been used to replicate a defective member of the Reoviridae (mammalian orthoreovirus), in which a structural protein was eliminated from the viral genome $^{73}$. A DISC vaccine strain would exhibit many of the safety features of inactivated vaccines, while preserving the expression of viral proteins at the natural sites of infection, as observed with live vaccines. It remains to be determined what dose will be required to elicit protective immunity. The defective nature of the DISC vaccine strain would make it a safer class of vaccine than viable vaccine strains with respect to the 

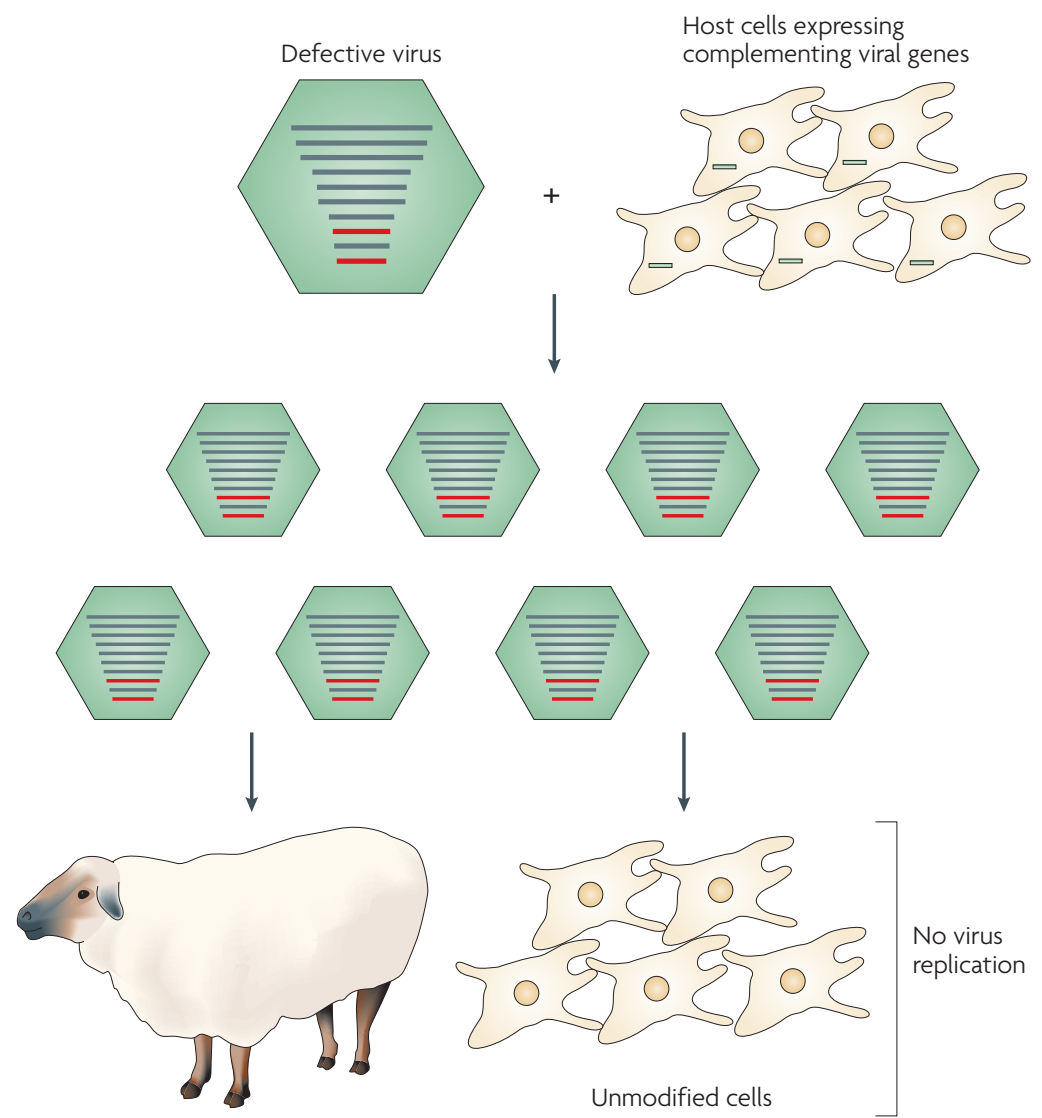

Animal
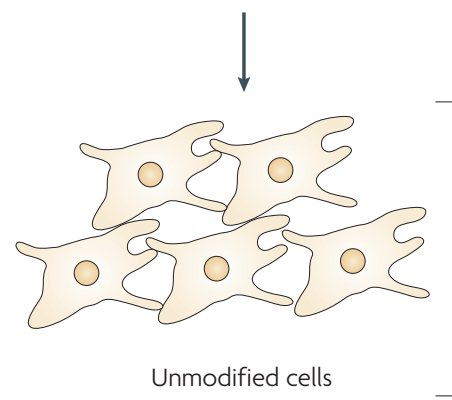

No virus replication

Figure 3 | Proposed DISC vaccine for bluetongue virus. Using the new reverse genetics system for bluetongue virus (BTV) it might be possible to make disabled infectious single cycle (DISC) vaccines for the virus. In these vaccines, multiple essential viral genes would be inactivated in the virus and supplied during vaccine production using a complementing cell line. In unmodified cells, and in the vaccinated animal, the virus would be unable to replicate because the complementing proteins would be missing. To ensure that progeny virions produced through recombination with field strains were non-viable, the DISC strain could be further attenuated using codon bias mutations in all ten segments. Such vaccines would be compatible with current vaccine-production facilities used to make attenuated and inactivated vaccines.

\section{Adjuvant}

A substance that is mixed with an immunogen to elicit a more marked immune

response in an organism risk of reversion to virulence. Because the DISC strain would be missing one or more viral proteins, the DISC approach could be used to make a vaccine in which it is possible to distinguish between vaccinated animals and those that have been exposed to infectious virus. Such information would permit an assessment on whether a country or region is bluetongue-free, a decision that has important consequences for the trade of livestock. A complementary strategy would be to use the suboptimal codon approach recently pioneered for polio vaccines ${ }^{74,75}$. This method alters the codon bias throughout the genome to attenuate the virus, and would effectively remove the possibility that, similar to current attenuated vaccines, the DISC strain might reassort with circulating field strains to produce viable virus. Reassortment between the codon-disabled DISC strain and a field strain would lead to progeny virus that would inherit the poor protein expression of the DISC parent irrespective of which genome segment were to reassort. As the entire genome of BTV is composed of only 19,219 nucleotides, this approach should be feasible.

\section{Concluding remarks}

The control of bluetongue disease through timely and relevant vaccination is feasible. However, although current vaccines are effective, they have significant drawbacks that are likely to increase as the demand for vaccination grows. The speed of scale up to a newly emerged serotype, the uncertain nature of natural attenuation and the safety issues associated with virus inactivation all suggest that newer approaches are needed. The new generation of vaccines described above offers amelioration for each of these areas by offering a rapid route from DNA to vaccine as well as a safe product without loss of a robust immune response. Such vaccines are at an advanced stage of development and it seems likely that some will find their way to the marketplace in the near future. The basis of BTV serotype and neutralization is known, and VP2-only (and perhaps other immunogenic BTV proteins) or VLP-based subunit vaccines could be beneficially applied to control the outbreak of bluetongue disease. DISC vaccines and codon bias vaccines for BTV represent an exciting future possibility, as they should allow increased safety with even better immunogenicity. In short, recombinant approaches are poised to become the dominant method for BTV vaccine development in the future. We look forward to the addition of molecular adjuvants and development of the BTV vaccine as a carrier for other vaccine antigens. Recombinant vaccines developed in this way could allow the introduction of a marker that could be used to distinguish between vaccinated and infected animals, a long sought after goal that would allow the safe movement of animals without the danger of introducing bluetongue disease.
1. Spreull, J. Malarial catarrhal fever (bluetongue) of sheep in South Africa. J. Comp. Pathol. Ther. 18, 321-337 (1905).

2. Hutcheon, D. Fever of epizootic catarrh. Rep. Coll. Vet. Surg. 1880, 12-15 (1881).

3. Elbers, A. R. et al. Field observations during the bluetongue serotype 8 epidemic in 2006. II. Morbidity and mortality rate, case fatality and clinical recovery in sheep and cattle in the Netherlands. Prev. Vet. Med. 87, 31-40 (2008).

4. Erasmus, B. J. Bluetongue in sheep and goats. Aust. Vet. J. 51, 165-170 (1975).

5. Erasmus, B. J. The control of bluetongue in an enzootic situation. Aust. Vet. J. 51, 209-210 (1975)
6. De Clercq, K. et al. Transplacental infection and apparently immunotolerance induced by a wild-type bluetongue virus serotype 8 natura infection. Transbound Emerg. Dis. 55, 352-359 infection. Transbound Emerg. Dis. 55, 352-359
(2008).

7. Menzies, F. D. et al. Evidence for transplacental and contact transmission of bluetongue virus in cattle. Vet. Rec. 163, 203-209 (2008).

8. Vercauteren, G. et al. Bluetongue virus serotype 8 -associated congenital hydranencephaly in calves Transbound Emerg. Dis. 55, 293-298 (2008).

9. Waldvogel, A. S., Anderson, G. A., Phillips, D. L. \& Osburn, B. I. Association of virulent and avirulent strains of bluetongue virus serotype 11 with premature births of late-term bovine fetuses J. Comp. Pathol. 106, 333-340 (1992)

10. Waldvogel, A. S., Anderson, G. A., Phillips, D. L. \& Osburn, B. I. Infection of bovine fetuses at 120 days' gestation with virulent and avirulent strains of bluetongue virus serotype 11. Comp. Immunol. Microbiol. Infect. Dis. 15, 53-63 (1992).

11. Purse, B. V. et al. Climate change and the recent emergence of bluetongue in Europe. Nature Rev. Microbiol. 3, 171-181 (2005).

12. Mellor, P. S., Carpenter, S., Harrup, L., Baylis, M. \& Mertens, P. P. Bluetongue in Europe and the Mediterranean Basin: history of occurrence prior to 2006. Prev. Vet. Med. 87, 4-20 (2008). 
13. Maan, S. et al. Sequence analysis of bluetongue virus serotype 8 from the Netherlands 2006 and comparison to other European strains. Virology 377, 308-318 (2008)

14. Mintiens, K. et al. Possible routes of introduction of bluetongue virus serotype 8 into the epicentre of the 2006 epidemic in north-western Europe. Prev. Vet. Med. 87, 131-144 (2008)

15. Gloster, J., Mellor, P. S., Manning, A. J., Webster, H. N. \& Hort, M. C. Assessing the risk of windborne spread of bluetongue in the 2006 outbreak of disease in northern Europe. Vet. Rec. 160, 54-56 (2007).

16. Roy, P. \& Noad, R. Bluetongue virus assembly and morphogenesis. Curr. Top. Microbiol. Immunol. 309 87-116 (2006).

17. Forzan, M., Marsch, M. \& Roy, P. Bluetongue virus entry into the cells. J. Virol. 81, 4819-4827 (2007)

18. Roy, P. in Fields' Virology (eds Knipe, D. M. \& Howley, P. M.) 1975-1997 (Lippincott Williams \& Wilkins, New York, 2007)

19. Roy, P., Bishop, D. H., LeBlois, H. \& Erasmus, B. J. Long-lasting protection of sheep against bluetongue challenge after vaccination with virus-like particles: evidence for homologous and partial heterologous protection. Vaccine 12, 805-811 (1994).

20. Jeggo, M. H., Wardley, R. C. \& Taylor, W. P. Role of neutralising antibody in passive immunity to bluetongue infection. Res. Vet. Sci. 36, 81-86 (1984).

21. Jeggo, M. H., Wardley, R. C. \& Brownlie, J. A study of the role of cell-mediated immunity in bluetongue virus infection in sheep, using cellular adoptive transfer techniques. Immunology 52, 403-410 (1984).

22. Jeggo, M. H., Wardley, R. C. \& Brownlie, J. Importance of ovine cytotoxic T cells in protection against bluetongue virus infection. Prog. Clin. Biol. Res. 178, 477-487 (1985)

23. Stott, J. L., Barber, T. L. \& Osburn, B. I. Immunologic response of sheep to inactivated and virulent bluetongue virus. Am. J. Vet. Res. 46, 1043-1049 (1985).

24. Batten, C. A., Maan, S., Shaw, A. E., Maan, N. S. \& Mertens, P. P. A European field strain of bluetongue virus derived from two parental vaccine strains by genome segment reassortment. Virus Res. 137, 56-63 (2008)

25. Collisson, E. W., Barber, T. L., Gibbs, E. P. \& Greiner E. C. Two electropherotypes of bluetongue virus serotype 2 from naturally infected calves. J. Gen. Virol. 66, 1279-1286 (1985).

26. Kahlon, J., Sugiyama, K. \& Roy, P. Molecular basis of bluetongue virus neutralization. J. Virol. 48 627-632 (1983).

27. Oberst, R. D., Squire, K. R., Stott, J. L., Chuang, R. Y. $\&$ Osburn, B. I. The coexistence of multiple bluetongue virus electropherotypes in individual cattle during natural infection. J. Gen. Virol. 66 1901-1909 (1985).

28. Oberst, R. D., Stott, J. L., Blanchard-Channell, M. \& Osburn, B. I. Genetic reassortment of bluetongue virus serotype 11 strains in the bovine. Vet. Microbiol. 15, 11-18 (1987).

29. Ramig, R. F., Garrison, C. Chen, D. \& Bell-Robinson, D. Analysis of reassortment and superinfection during mixed infection of Vero cells with bluetongue virus serotypes 10 and 17. J. Gen. Virol. 70 2595-2603 (1989).

30 Samal S. K., Livingston, C. W. Jr. McConnell, S. \& Ramig, R. F. Analysis of mixed infection of sheep with bluetongue virus serotypes 10 and 17: evidence for genetic reassortment in the vertebrate host. J. Virol. 61, 1086-1091 (1987).

31. Sugiyama, K., Bishop, D. H. \& Roy, P. Analyses of the genomes of bluetongue viruses recovered in the United States. I. Oligonucleotide fingerprint studies that indicate the existence of naturally occurring reassortant BTV isolates. Virology 114, 210-217 (1981).

32. Theiler, A. in Ann. Rept. Director Agric. Transvaal for 1904-1905. 110-121 (1906).

33. Alexander, R. A. \& Haig, D. A. The use of egg attenuated bluetongue virus in the production of a polyvalent vaccine for sheep: a propagation of the virus in sheep. Onderstepport J. Vet. Sci. Anim. Ind. 25, 3-15 (1951)

34. Hunter, P. \& Modumo, J. A monovalent attenuated serotype 2 bluetongue virus vaccine confers homologous protection in sheep. Onderstepoort J. Vet. Res. 68, 331-333 (2001).
35. Lacetera, N. \& Ronchi, B. Evaluation of antibody response and nonspecific lymphocyte blastogenesis following inoculation of a live attenuated bluetongue virus vaccine in goats. Am. J. Vet. Res. 65 1331-1334 (2004)

36. Veronesi, E., Hamblin, C. \& Mellor, P. S. Live attenuated bluetongue vaccine viruses in Dorset Poll sheep, before and after passage in vector midges (Diptera: Ceratopogonidae). Vaccine $\mathbf{2 3}$ 5509-5516 (2005).

37. Savini, G. et al. Assessment of efficacy of a bivalent BTV-2 and BTV-4 inactivated vaccine by vaccination and challenge in cattle. Vet. Microbiol. 133, 1-8 (2008)

38. Schultz, G. \& Delay, P. D. Losses in newborn lambs associated with bluetongue vaccination of pregnancy ewes. J. Am. Vet. Med. Assoc. 127, 224-226 (1955)

39. Young, S. \& Cordy, D. R. An ovine fetal encaphalopathy caused by bluetongue virus vaccine. J. Neuropathol Exp. Neurol. 23, 635-642 (1964).

40. Osburn, B. I. et al. Experimental viral-induced congenital encephalopathies. II. The pathogensis of bluetongue virus infection of fetal lambs. Lab. Invest. 25, 206-213 (1971)

41. Ferrari, G. et al. Active circulation of bluetongue vaccine virus serotype- 2 among unvaccinated cattle in central Italy. Prev. Vet. Med. 68, 103-113 (2005)

42. Stott, J. L., Oberst, R. D., Channell, M. B. \& Osburn, B. I. Genome segment reassortment between two serotypes of bluetongue virus in a natural host. J. Virol. 61, 2670-2674 (1987).

43. Batten, C. A., Maan, S., Shaw, A. E., Maan, N. S. \& Mertens, P. P. A European field strain of bluetongue virus derived from two parental vaccine strains by genome segment reassortment. Virus Res. 137 56-63 (2008).

44. Parker, J., Herniman, K. A., Gibbs, E. P. \& Sellers, R. F. An experimental inactivated vaccine against bluetongue. Vet. Rec. 96, 284-287 (1975).

45. Savini, G. et al. An inactivated vaccine for the control of bluetongue virus serotype 16 infection in sheep in Italy. Vet. Microbiol. 124, 140-146 (2007).

46. Campbell, C. H. Immunogenicity of bluetongue virus inactivated by gamma irradiation. Vaccine 3 401-406 (1985)

47. Berry, L. J. et al. Inactivated bluetongue virus vaccine in lambs: differential serological responses related to breed. Vet. Res. Commun. 5, 289-293 (1982).

48. Ramakrishnan, M. A. et al. Immune responses and protective efficacy of binary ethylenimine (BEI)inactivated bluetongue virus vaccines in sheep. Vet Res. Commun. 30, 873-880 (2006).

49. Savini, G., MacLachlan, N. J., Sanchez-Vizcaino, J. M. \& Zientara, S. Vaccines against bluetongue in Europe. Comp. Immunol. Microbiol. Infect. Dis. 31 101-120 (2008)

50. Huismans, H., Van der Walt, N. T., Cloete, M. \& Erasmus, B. J. Isolation of a capsid protein of bluetongue virus that induces a protective immune response in sheep. Virology 157, 172-179 (1987).

51. Roy, P., Urakawa, T., Van Dijk, A A \& Erasmus, B. Recombinant virus vaccine for bluetongue disease in sheep. J. Virol. 64, 1998-2003 (1990)

52. Lobato, Z. I., Coupar, B. E., Gray, C. P., Lunt, R. \& Andrew, M. E. Antibody responses and protective immunity to recombinant vaccinia virus-expressed bluetongue virus antigens. Vet. Immunol. Immunopathol. 59, 293-309 (1997).

53. Boone, J. D. et al. Recombinant canarypox virus vaccine co-expressing genes encoding the VP2 and VP5 outer capsid proteins of bluetongue virus induces high level protection in sheep. Vaccine 25 672-678 (2007).

54. Perrin, A. et al. Recombinant capripoxviruses expressing proteins of bluetongue virus: evaluation of immune responses and protection in small ruminants. Vaccine 25, 6774-6783 (2007).

55. Noad, R. \& Roy, P. Virus-like particles as immunogens. Trends Microbiol. 11, 438-444 (2003)

56. Roy, P. \& Noad, R. Virus-like particles as a vaccine delivery system: myths and facts. Hum. Vaccin. 4 5-12 (2008)

57. Harper, D. M. et al. Sustained efficacy up to 4.5 years of a bivalent $L 1$ virus-like particle vaccine against human papillomavirus types 16 and 18 : follow-up from a randomised control trial. Lancet 367, 1247-1255 (2006).
58. Barr, E. \& Tamms, G. Quadrivalent human papillomavirus vaccine. Clin. Infect. Dis. $\mathbf{4 5}$ 609-617 (2007)

59. Franco, E. L. \& Harper, D. M. Vaccination against human papillomavirus infection: a new paradigm in cervical cancer control. Vaccine 23, 2388-2394 (2005).

60. Harper, D. M. et al. Efficacy of a bivalent L1 virus like particle vaccine in prevention of infection with human papillomavirus types 16 and 18 in young women: a randomised controlled trial. Lancet 364 1757-1765 (2004)

61. French, T. J., Marshall, J. J. \& Roy, P. Assembly of double-shelled, virus-like particles of bluetongue virus by the simultaneous expression of four structural proteins. J. Virol. 64, 5695-5700 (1990).

62. Possee, R. D., Thomas, C. J. \& King, L. A. The use of baculovirus vectors for the production of membrane proteins in insect cells. Biochem. Soc. Trans. 27 928-932 (1999).

63. French, T. J. \& Roy, P. Synthesis of bluetongue virus (BTV) corelike particles by a recombinant baculovirus expressing the two major structural core proteins of BTV. J. Virol. 64, 1530-1536 (1990).

64. Rose, R. C., Bonnez, W., Reichman, R. C. \& Garcea, R. L. Expression of human papillomavirus type $11 \mathrm{~L} 1$ protein in insect cells: in vivo and in vitro assembly of viruslike particles. J. Virol. 67, 1936-1944 (1993).

65. Hewat, E. A., Booth, T. F., Loudon, P. T. \& Roy, P. Three-dimensional reconstruction of baculovirus expressed bluetongue virus core-like particles by cryo-electron microscopy. Virology 189, 10-20 (1992).

66. Hewat, E. A., Booth, T. F. \& Roy, P. Structure of correctly self-assembled bluetongue virus-like particles. J. Struct. Biol. 112, 183-191 (1994).

67. Prasad, B. V V. Yamaguchi, S. \& Roy, P. Threedimensional structure of single-shelled bluetongue virus. J. Virol. 66, 2135-2142 (1992)

68. Nason, E. et al. Interactions between the inner and outer capsids of bluetongue virus. J. Virol. 78, 8059-8067 (2004).

69. Loudon, P. T., Hirasawa, T., Oldfield, S., Murphy, M. $\&$ Roy, P. Expression of the outer capsid protein VP5 of two bluetongue viruses, and synthesis of chimeric double-shelled virus-like particles using combinations of recombinant baculoviruses. Virology 182, 793-801 (1991).

70. Roy, P., French, T. \& Erasmus, B. J. Protective efficacy of virus-like particles for bluetongue disease. Vaccine 10, 28-32 (1992).

71. Roy, P. in Vaccines for OIE List A and Emerging Animal Diseases (eds Roth, J. \& Brown, F.) 113-147 (Karger, Basel, 2002)

72. Boyce, M., Celma, C. P. \& Roy, P. Development of a reverse genetics system for bluetongue virus: recovery of infectious virus from synthetic RNA transcripts. J. Virol. 82, 8339-8348 (2008).

73. Roner, M. R. \& Joklik, W. K. Reovirus reverse genetics: incorporation of the CAT gene into the reovirus genome. Proc. Natl Acad. Sci. USA 98 8036-8041 (2001).

74. Coleman, J. R. et al. Virus attenuation by genomescale changes in codon pair bias. Science 320 1784-1787 (2008)

75. Burns, C. C. et al. Modulation of poliovirus replicative fitness in HeLa cells by deoptimization of synonymous codon usage in the capsid region. J. Virol. 80, 3259-3272 (2006)

DATABASES

UniProtKB: http://www.uniprot.org

$\underline{\mathrm{NS} 1}|\underline{\mathrm{NS} 3}| \underline{\mathrm{VP1}}|\mathrm{VP2}| \underline{\mathrm{VP} 3}|\underline{\mathrm{VP} 4}| \underline{\mathrm{VP} 5}|\underline{\mathrm{VP} 6}| \mathrm{VP7}$

FURTHER INFORMATION

Polly Roy's homepage: http://www.lshtm.ac.uk/pmbu/

research/roylab

The Center for Food Security and Public Health (animal disease information): http://www.cfsph.iastate.edu/ diseaseinfo/

National Farmers Union: http://www.nfuonline.com/ $\times 32254 . x m l$

World Organisation for Animal Health (bluetongue diseases data): http://www.oie.int/eng/maladies/fiches/a A090.htm\#4.

ALL LINKS ARE ACTIVE IN THE ONLINE PDF 